ARID International Journal for Science and Technology (AIJST)

ISSN: 2662-009X

Journal home page: http://arid.my/j/aijst

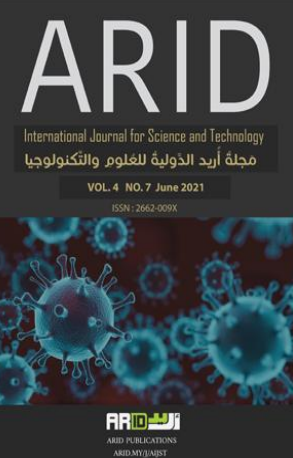

$$
\text { مَجلةُ أُريد الدَّوليةُ للعُلورج والتّكنولوجيا }
$$

\title{
UNION AND INTERSECTION OF CHAOTIC GRAPHS WITH DENSITY VARIATION
}

Fathia Milad Alogab Ibtesam Milad Laqab

Mathematics department - Science College - Al-Asmarya Islamic University - Libya

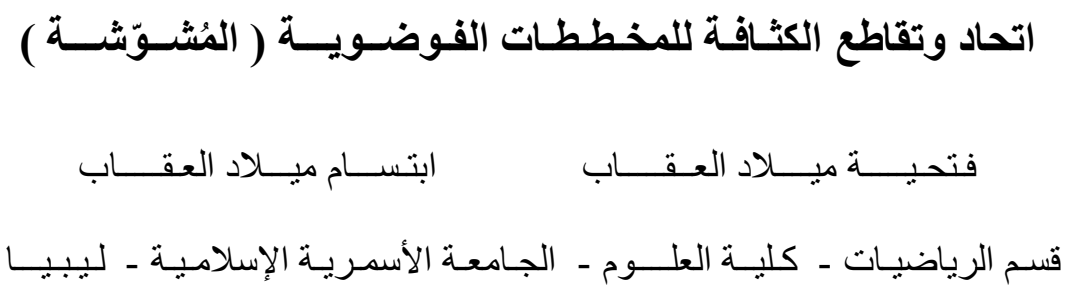

fathiaalagab@gmail.com

arid.my/0003-9675

https://doi.org/10.36772/arid.aijst.2021.471 


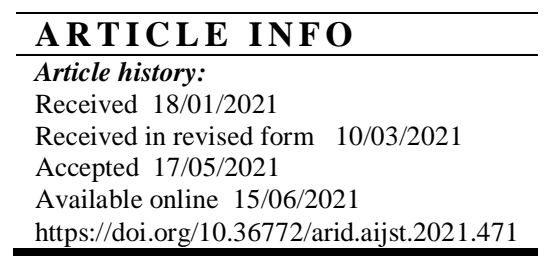

\begin{abstract}
The aim of the present study is to discuss the union and intersection operations on chaotic graphs with density variation; the adjacency and incidence matrices representing the chaotic graphs induced from these operations will be introduced when physical characters of chaotic graphs have the same properties.

There are several applications that have been utilized on chaotic graphs with density variation. The most practical applications of these kinds of operations on chaotic graphs with density variation are the internet signal speeds and the variation of green color for different parts of the plant. For example, in botany, in some cases several plants suffer from a lack of chlorophyll in the damaged parts of the plant. In this case the plant is represented by a chaotic graph and the proportion of chlorophyll is represented by the density property, then the appropriate process is applied to increase the chlorophyll percentage in the appropriate place, so these operations helps us to choose the suitable operator that satisfy our desires and requests.
\end{abstract}

Keywords: adjacency matrix, incidence matrix, chaotic graph, density, union, intersection. 


\section{المـلخـص}

في هذا البحث سوف يتم مناقشة بعض العمليات الجبرية على المخططات الفوضوية ( المُشُّشّة ) التي تحمل خاصية الكثافة وهما

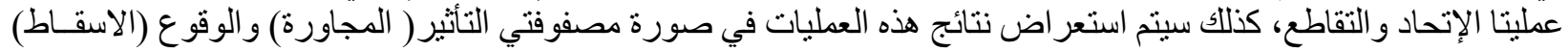
عندما يكون المخطط المشوش يحمل خاصية والنية واحدة.

هذا البحث يناقش تأثير هذه العمليات على مستوى الكثافة لدى المخططات المشوّشة (الفوضوية)، حيث يمكننا تمثيل خاصية الكثافة الكئة

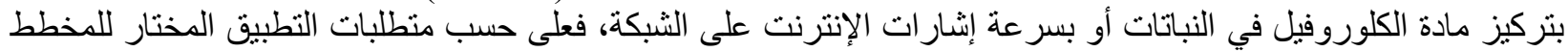

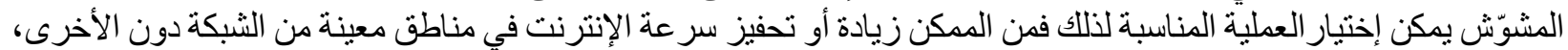

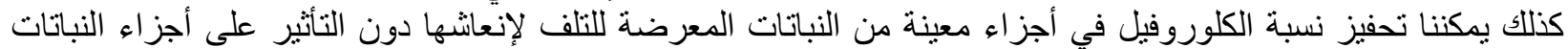

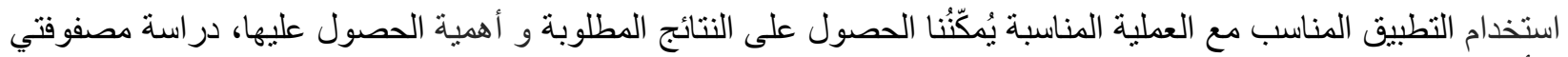

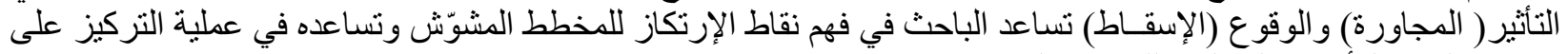

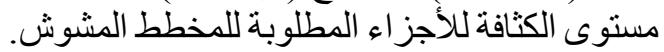

الكلمـات المفتاحيـة: مصفوفة التأثير (المجاورة)، مصفوفة الوقوع (الإسقاط)، المخطط الفوضوي (المشوش)، كثافة، إتحاد، تقاطع. 


\section{INTRODUCTION}

Scientists of all physical systems are searching for a simple structure that describe the topology of the system in a simple way which help them to develop the performance of the system, the theory of chaotic graphs is introduced to describe most of these physical systems, it visualizes the topology of the system, so scientist can apply their physical character on the system and understand the behavior of the physical character. In this research, the physical character is presented by the density character; the density character can represent many physical applications in different fields such as biology fields (i.e. Chlorophyll degree, Bacterial grows, nerve system), chemical systems (i.e. atoms topology), internet technology (i.e. web internet signal speed) and many other different fields. [1, 2]

Our interest is applying the algebraic part of the chaotic graph and translated on the chosen applications, so the theory of chaotic graphs can help scientists to understand and control their applications.

Basic topological definitions and backgrounds are relevant to this article are cited in this background.

\subsection{Definitions and backgrounds}

- Simple graph $G(V, E)$ : Is a graph with no loops or multiple edges [3].

- Adjacency and incidence: Let $v$ and $w$ be vertices of a graph, if $v$ and $w$ are joined by an edge $e$, then $v$ and $w$ are said to be adjacent. Moreover, $v$ and $w$ are said to be incident with $e$, and $e$ is said to be incident with $v$ and $w[4,5,6]$.

- The adjacency matrix: Let $G$ be a graph without loops, with $n$-vertices labeled $1,2,3, \ldots, n$ the "adjacency matrix" $A(G)$ is the $n \times n$ matrix in which the entry in row $i$ and column $j$ is the number of edges joining the vertices $i$ and $j[4,5,6]$. 
- The incidence matrix: Let $G$ be a graph without loops, with $n$-vertices labeled $1,2,3, \ldots, n$ and $m$ - edges labeled $1,2,3, \ldots, m$.The" incidence matrix" $I(G)$ is the $n \times m$ matrix in which the entry in row $i$ and column $j$ is 1 if vertex $i$ is incident with edge $j$ and 0 otherwise $[4,5,6]$.

Example: Consider the graph $G$ in Figure (1):

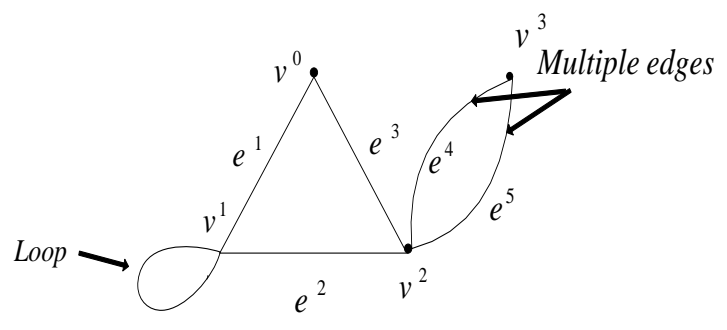

Figure (1): Multiple graph

The adjacency matrix $A(G)$ is: $A(G)=\left[\begin{array}{llll}0 & 1 & 1 & 0 \\ 1 & 1 & 1 & 0 \\ 1 & 1 & 0 & 2 \\ 0 & 0 & 2 & 0\end{array}\right]$

And its incidence matrix $I(G)$ is: $I(G)=\left[\begin{array}{ccccc}1 & 0 & 1 & 0 & 0 \\ 1^{1} & 1 & 0 & 0 & 0 \\ 0 & 1 & 1 & 1 & 1 \\ 0 & 0 & 0 & 1 & 1\end{array}\right]$

Noting that the symbol ( $\left.1^{1}\right)$ in the second row and first column means that we have one loop at the vertex $v^{1}$ with the edge $e^{1}$. Also, if we have two loops at the same above vertex, we symbolize it by $1^{11}$. Moreover, if we have an infinite number of loops at any vertex, say $v^{i}$, we use the symbol $1^{111 \ldots}$ to represent them [1].

- Chaotic graph $G_{h}\left(V_{h}, E_{h}\right)$ : Is a geometric graph that carries many physical characters, these geometric graphs might have similar properties or different $[5,7,8,9]$. 
- Null graph: Is a graph consists of a set of vertices and no edges $[9,10]$.

- Loop: A loop is an edge which starts and ends on the same vertex $[6,7,8]$.

- Multiple edges: Tow or more edges joining the same pair of vertices are called "multiple edges" [2].

- Density (d): Is a physical property of matter, as each element and compound has a unique density associated with it [11].

- The "union" $G$ of two graphs $G_{1}$ and $G_{2}$ is a graph $G=G_{1} \cup G_{2}$ having $V(G)=V\left(G_{1}\right) \bigcup V\left(G_{2}\right)$ and $E(G)=E\left(G_{1}\right) \bigcup E\left(G_{2}\right)$ such that $V\left(G_{1}\right) \bigcap V\left(G_{2}\right)=\phi[10]$.

- The "intersection" of two graphs $G_{1}=\left(V_{1}, E_{1}\right)$ and $G_{2}=\left(V_{2}, E_{2}\right)$, with at least one vertex in common than their intersection will be the graph of vertices and edges defined as follows: $V\left(G_{1} \cap G_{2}\right)=V\left(G_{1}\right) \cap V\left(G_{2}\right)$ and $E\left(G_{1} \cap G_{2}\right)=E\left(G_{1}\right) \cap E\left(G_{2}\right)$ [9].

\subsection{The matrix representation for fixed and different densities of chaotic graphs:}

In this part we will obtain the adjacent and incidence matrices for two cases:

1. When degree of density is fixed, for example degree of green color of a leave is a perfect green.

2. Degree of density is different and various between chaotic levels and areas. For example degree of green color of a leave is various between different parts of a leave.

We will denote the degree of each area on the chaotic graph by $d_{p q}$, where $p$ denotes level of chaotic graph, while $q$ denotes different areas on each level of chaotic graph [11] .See Figure (2) 


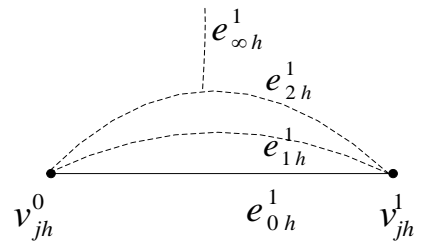

Figure (2): 1-Simplicial chaotic graph

And the adjacent and incidence matrices are:

$$
A\left(G_{h}\right)=\left[\begin{array}{ll}
0_{(0123 . . \infty)_{d p q} h} & 1_{(0123 \ldots \infty)_{d p q} h} \\
1_{(0123 \ldots)_{d p q} h} & 0_{(0123 \ldots \infty)_{d p q} h}
\end{array}\right], I\left(G_{h}\right)=\left[\begin{array}{c}
1_{(0123 . \ldots)_{d p q} h} \\
1_{(0123 \ldots \infty)_{d p q} h}
\end{array}\right]
$$

\section{Union and intersection of chaotic graphs with density variations:}

\subsection{Union of chaotic graphs with density variation}

This part will discuss and explain the union of two chaotic graphs with density variation, we shall show the union of two simple and multiple chaotic graphs inducing the adjacent and incidence matrices of the resulted, discussion can be understand it clearly via examples.

Consider two simple chaotic graphs with density variation $G_{h}{ }^{1}$ and $G_{h}{ }^{2}$ which are $G_{h}^{1}=\left\{v_{j h}^{0} e_{0 h}^{1} v_{j h}^{1} ; j=0,1,2 \ldots \infty\right\}, G_{h}^{2}=\left\{v_{j h}^{2} e_{j h}^{2} v_{j h}^{3} e_{j h}^{3} v_{j h}^{4} e_{j h}^{4} v_{j h}^{2} ; j=0,1,2, \ldots \infty\right\}$ and their union $G_{h}^{1} \cup G_{h}^{2}=\left\{v_{j h}^{0} e_{j h}^{1} v_{j h}^{1} v_{j h}^{2} e_{j h}^{2} v_{j h}^{3} e_{j h}^{3} v_{j h}^{4} e_{j h}^{4} v_{j h}^{2} ; j=0,1,2, \ldots \infty\right\}$ is shown in Figure (3)
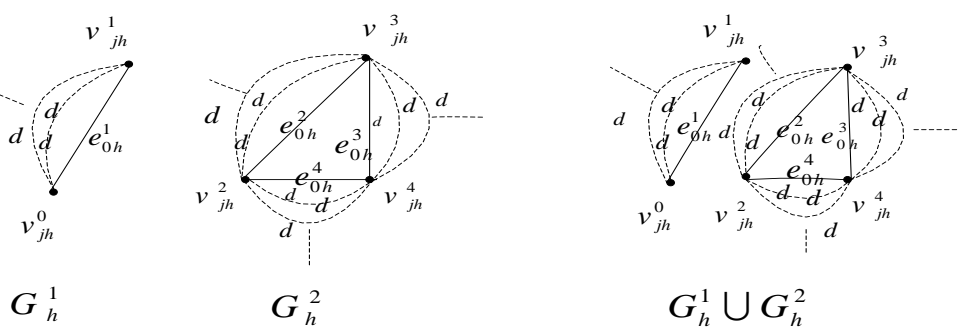

Figure (3): Union of two simple chaotic graphs 
Both the adjacent and incidence matrices of $G_{h}^{1}, G_{h}^{2}$ and $G_{h}^{1} \cup G_{h}^{2}$ respectively are:

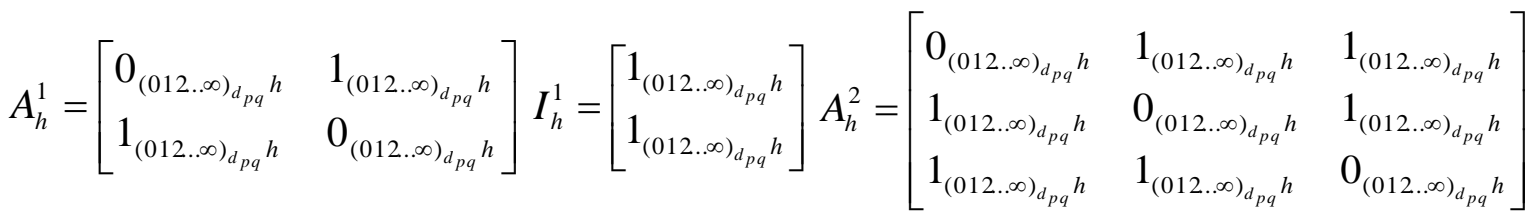

$$
\begin{aligned}
& I_{h}^{2}=\left[\begin{array}{lll}
1_{(012 \ldots \infty)_{d p q} h} & 0_{(012 \ldots \infty)_{d p q} h} & 1_{(012 \ldots \infty)_{d p q}} h \\
1_{(012 . . \infty)_{d p q} h} & 1_{(012 \ldots \infty)_{d p q} h} & 0_{(012 \ldots \infty)_{d p q}} h \\
0_{(012 \ldots)_{d p q} h} & 1_{(012 \ldots \infty)_{d p q} h} & 1_{(012 \ldots \infty)_{d p q} h}
\end{array}\right]
\end{aligned}
$$

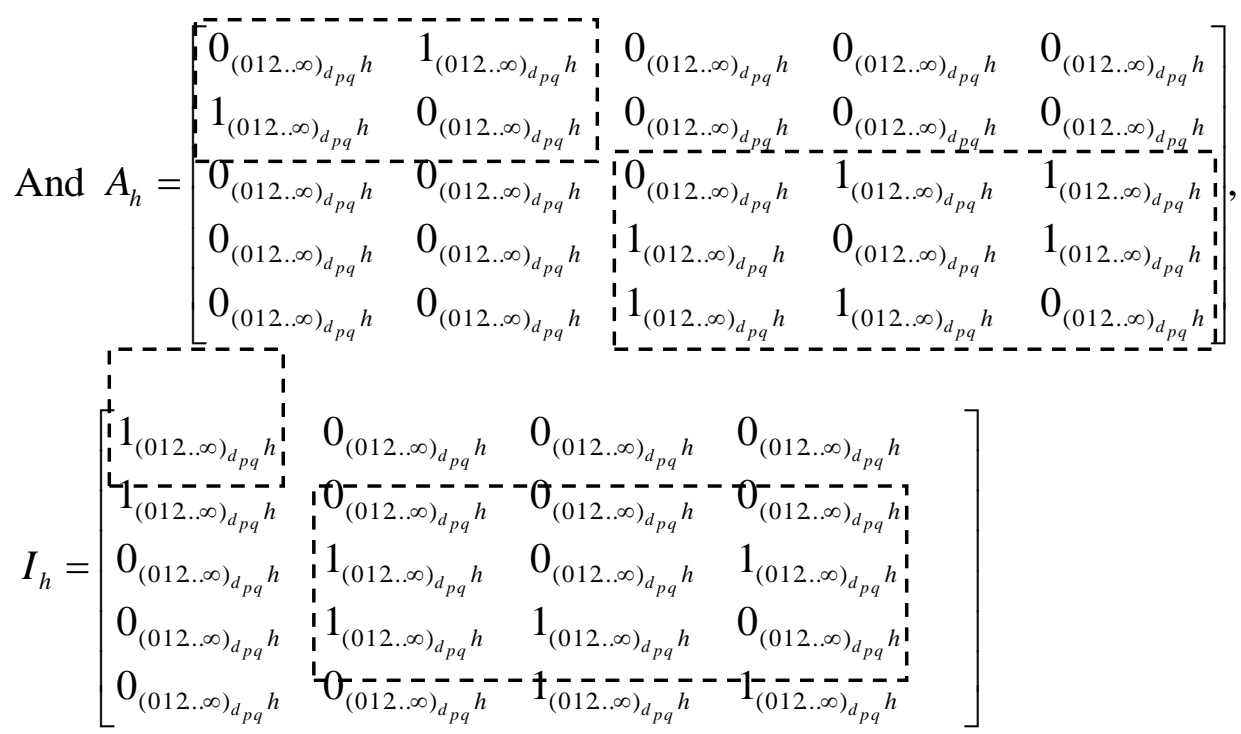

As the above result the total density of the resulted graph is increased.

Consider two multiple chaotic graphs with density variation $G_{h}^{1}\left(V_{h}^{1}, E_{h}^{1}\right)$ and $G_{h}^{2}\left(V_{h}^{2}, E_{h}^{2}\right)$ such that $V_{h}^{1}=\left\{v_{j h}^{0}, v_{j h}^{1} ; j=0,1,2 \ldots \infty\right\} E_{h}^{1}=\left\{e_{j h}^{1} ; j=0,1,2 \ldots \infty\right\}, V_{h}^{2}=\left\{v_{j h}^{2}, v_{j h}^{3}, v_{j h}^{4} ; j=0,1,2 \ldots \infty\right\}$ $E_{h}^{2}=\left\{e_{j h}^{2}, e_{j h}^{3}, e_{j h}^{4}, e_{j h}^{5} ; j=0,1,2 \ldots \infty\right\}$. See Figure (4) 


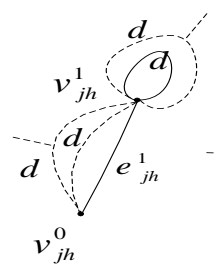

$G_{h}^{1}$

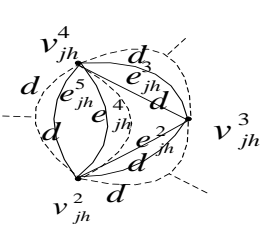

$G_{h}^{2}$

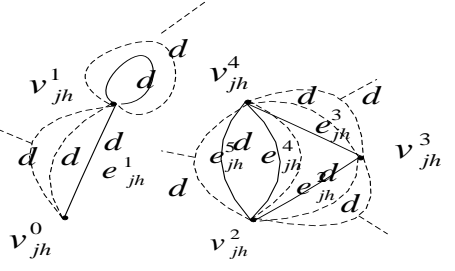

$G_{h}^{1} \cup G_{h}^{2}$

Figure (4): Union of two multiple chaotic graphs

Both the adjacent and incidence matrices of $G_{h}^{1}, G_{h}^{2}$ and $G_{h}^{1} \cup G_{h}^{2}$ respectively are:

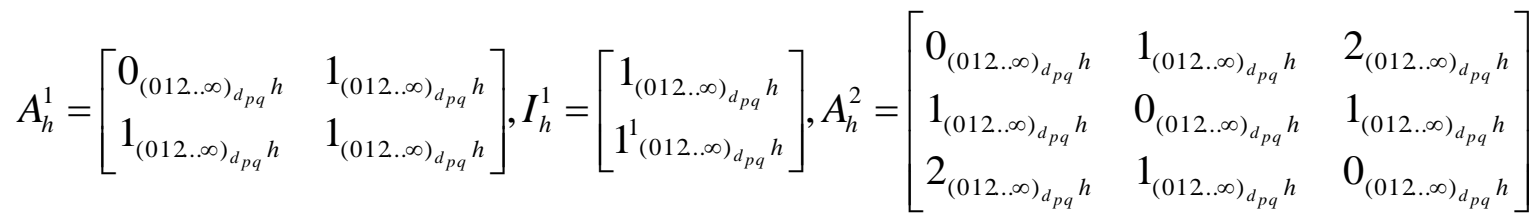

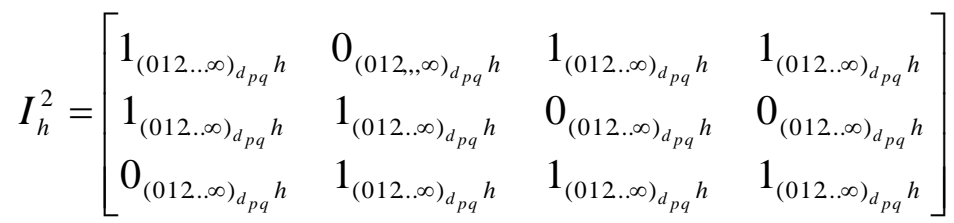

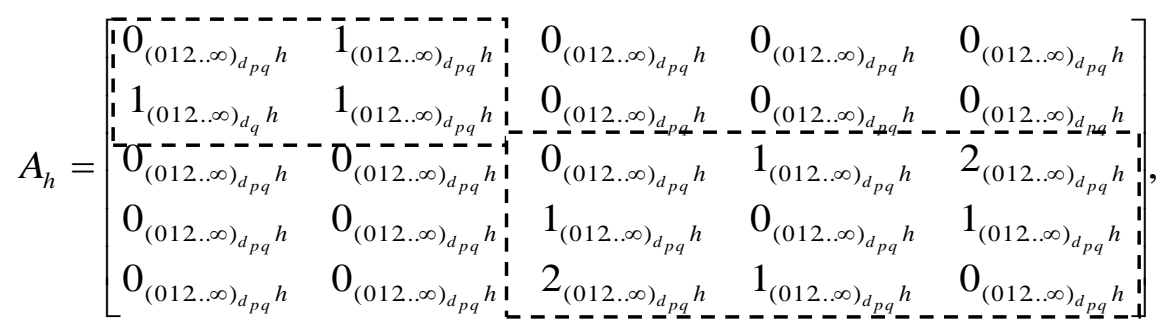

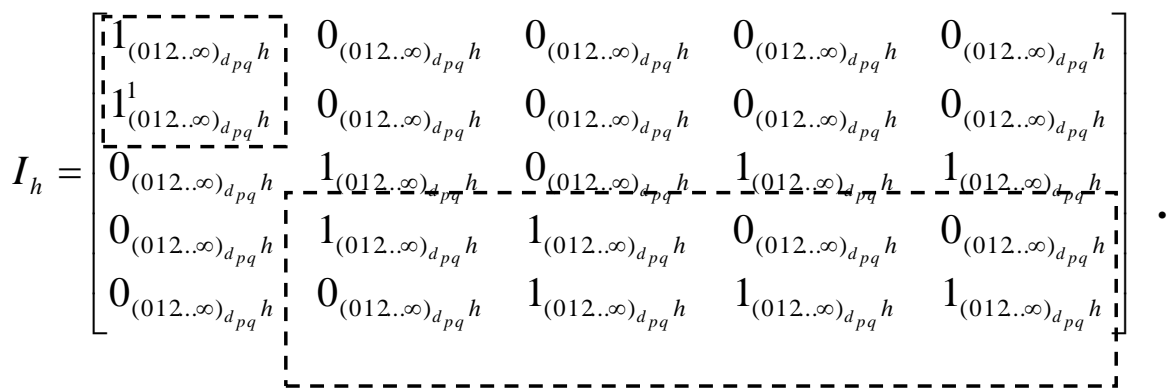


The union of two chaotic graphs with density variation is another chaotic graph with more extremer density value to the resulted chaotic graph. The adjacent and incidence matrices representing the union of two chaotic graphs can be obtained from the adjacent and incidence matrices of representing each of the given chaotic graphs constituting such a union of both of them by adding to them some chaotic zero matrices.

\subsection{Intersection of chaotic graphs with density variation:}

The operation of intersection on chaotic graphs is quite interesting compared to the operation of union; the following discussion explains the intersection of any two chaotic graphs with density variation by examples in several cases (i.e. disjoint, common vertices and common edges). The results of intersection of any two chaotic graphs are based on the intersection of any two geometric graphs.

\subsubsection{Disjoint intersection of chaotic graphs with density variation:}

Consider two simple chaotic graphs with density variation $G_{h}^{1}=\left\{v_{j h}^{0} e_{j h}^{1} v_{j h}^{1} ; j=0,1,2, \ldots\right\}$ and $G_{h}^{2}=\left\{v_{j h}^{2} e_{j h}^{2} v_{j h}^{3} e_{j h}^{3} v_{j h}^{4} ; j=0,1,2, \ldots\right\}$. See Figure (5)
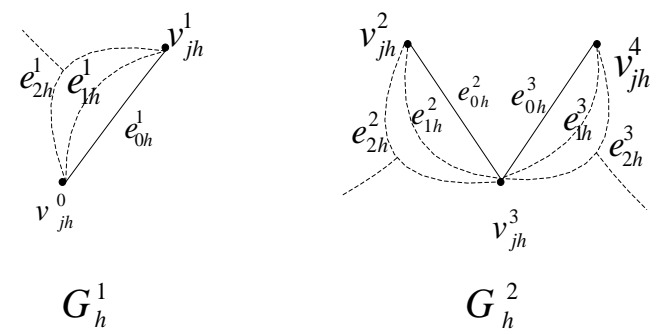

Figure (5): Two simple chaotic graphs 
Applying the intersection on $G_{h}^{1}$ and $G_{h}^{2}$, (i.e. $G_{h}^{1} \cap G_{h}^{2}$ ) results the empty graph, and both the adjacent and incidence matrices representing the chaotic graphs with density variation $G_{h}^{1}, G_{h}^{2}$ and $G_{h}^{1} \cap G_{h}^{2}$ are:

$$
\begin{aligned}
& A_{h}^{1}=\left[\begin{array}{ll}
0_{(012 . . \infty)_{d_{p q}} h} & 1_{(012 \ldots \infty)_{d p q}} h \\
1_{(012 . . \infty)_{d_{p q}} h} & 0_{(012 . . \infty)_{d p q} h}
\end{array}\right], I_{h}^{1}=\left[\begin{array}{c}
1_{(012 \ldots \infty)_{d p q}} h \\
1_{(012 . . \infty)_{d p q} h}
\end{array}\right],
\end{aligned}
$$

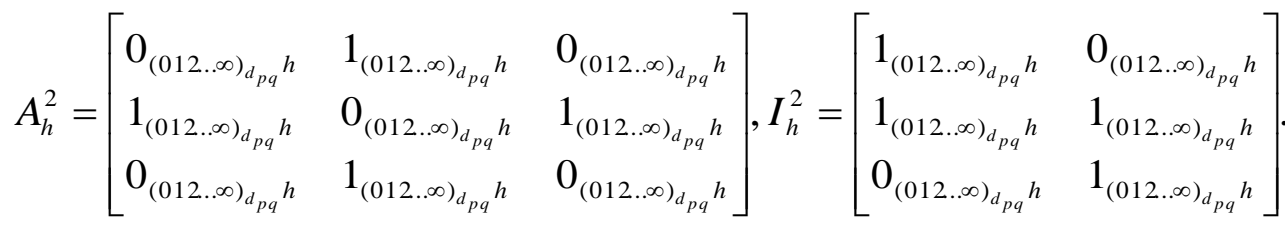

While the adjacent and incidence matrices of the intersected chaotic graph $G_{h}^{1} \cap G_{h}^{2}$ is the $\phi$ -matrix, (I.e. $A_{h}=\phi-$ matrix,$I_{h}=\phi$-matrix ).

So; if any two chaotic graphs with density variation are disjoint, then both of the adjacent and incidence matrices representing that intersection are $\phi$-matrix, and the density value of the intersected graph is zero.

\subsubsection{The intersection of two chaotic graphs with density variation common in one vertex} or more vertices:

Consider two simple chaotic graphs with density variation $G_{h}^{1}=\left\{v_{j h}^{0} e_{j h}^{1} v_{j h}^{1} ; j=0,1,2,3 \ldots\right\}$ and $G_{h}^{2}=\left\{v_{j h}^{0} e_{j h}^{2} v_{j h}^{1} e_{j h}^{3} v_{j h}^{2} ; j=0,1,2,3 \ldots\right\}$, then their intersection $G_{h}^{1} \cap G_{h}^{2}$ is the null graph of only two chaotic vertices $\left\{v_{j h}^{0}, v_{j h}^{1} ; j=0,1,2 \ldots\right\}$. See Figure (6). 

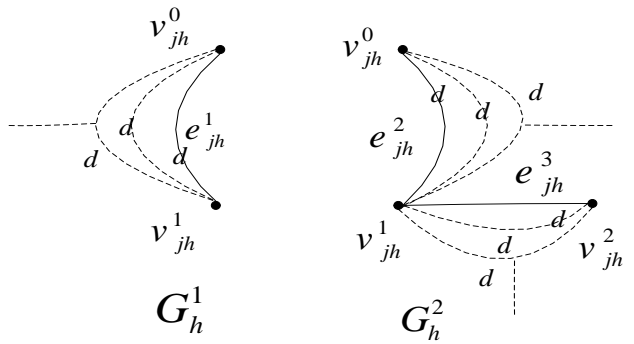

Figure (6): Two chaotic graphs common in two vertices

The density value in the intersected graph is greater than the original two chaotic graphs. The adjacent and incidence matrices of $G_{h}^{1}, G_{h}^{2}$ and $G_{h}^{1} \cap G_{h}^{2}$ are:

$$
\begin{aligned}
& A_{h}^{1}=\left[\begin{array}{ll}
0_{(012 . . \infty)_{d p q} h} & 1_{(012 \ldots \infty)_{d p q} h} \\
1_{(012 . . \infty)_{d_{p q}} h} & 0_{(012 . . \infty)_{d_{p q}} h}
\end{array}\right], I_{h}^{1}=\left[\begin{array}{c}
1_{(012 \ldots \infty)_{d p q}} h \\
1_{(012 \ldots \infty)_{d p q} h}
\end{array}\right],
\end{aligned}
$$

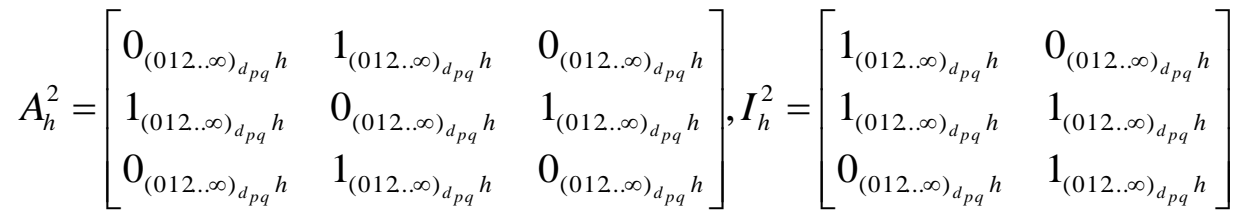

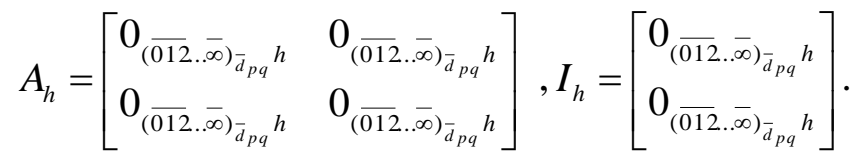

The adjacent and incidence matrices of the intersection of two chaotic vertices with density variation in the case if they are common in one vertex or many vertices and without any common edges are zeros matrices, but they have a double density in the intersected chaotic vertices.

\subsubsection{The intersection of two chaotic graphs with density variation common in more than one edge:}

Consider two simple chaotic graphs with density variation $G_{h}^{1}=\left\{v_{j h}^{0} e_{j h}^{1} v_{j h}^{1} e_{j h}^{2} v_{j h}^{2} e_{j h}^{3} v_{j h}^{3} ; j=0,1,2,3, \ldots\right\}$ and $G_{h}^{2}=\left\{v_{j h}^{1} e_{j h}^{2} v_{j h}^{2} e_{j h}^{3} v_{j h}^{3} e_{j h}^{4} v_{j h}^{4} ; j=0,1,2,3, \ldots\right\}$. See Figure 
The intersection of $G_{h}^{1} \cap G_{h}^{2}$ results another simple chaotic graph with denser value at these locations, and the adjacent and incidence matrices of $G_{h}^{1}, G_{h}^{2}$ and $G_{h}^{1} \cap G_{h}^{2}$ are:
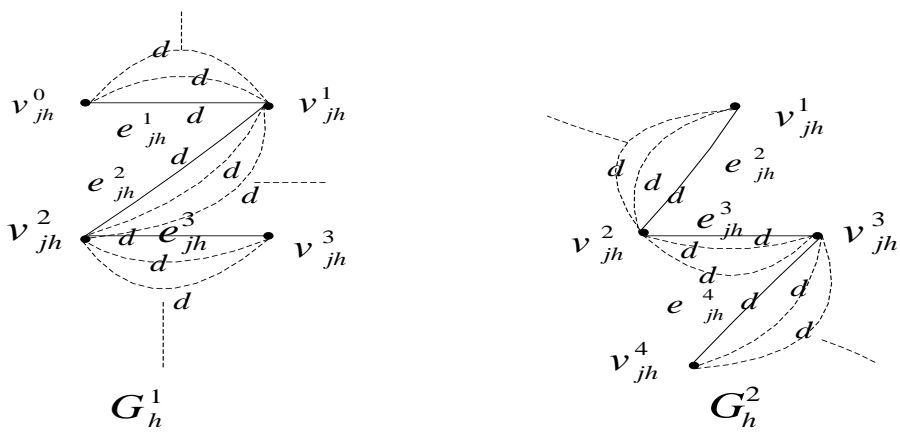

Figure (7): Intersection of two simple chaotic graphs common in two edges

$$
\begin{aligned}
& A_{h}^{1}=\left[\begin{array}{llll}
0_{(012 . . \infty)_{d_{p q}} h} & 1_{(012 . . \infty)_{d_{p q}} h} & 0_{(012 . . \infty)_{d_{p q}} h} & 0_{(012 . . \infty)_{d_{p q}} h} \\
1_{(012 . . \infty)_{d_{p q}} h} & 0_{(012 . . \infty)_{d_{p q}} h} & 1_{(012 . . \infty)_{d_{p q}} h} & 0_{(012 . . \infty)_{d_{p q}} h} \\
0_{(012 . . \infty)_{d_{p q}} h} & 1_{(012 . . \infty)_{d_{p q}} h} & 0_{(012 . . \infty)_{d_{p q}} h} & 1_{(012 . . \infty)_{d_{p q}} h} \\
0_{(012 . . \infty)_{d_{p q}} h} & 0_{(012 . . \infty)_{d p q} h} & 1_{(012 . . \infty)_{d_{p q}} h} & 0_{(012 . . \infty)_{d_{p q}} h}
\end{array}\right]=A_{h}^{2},
\end{aligned}
$$

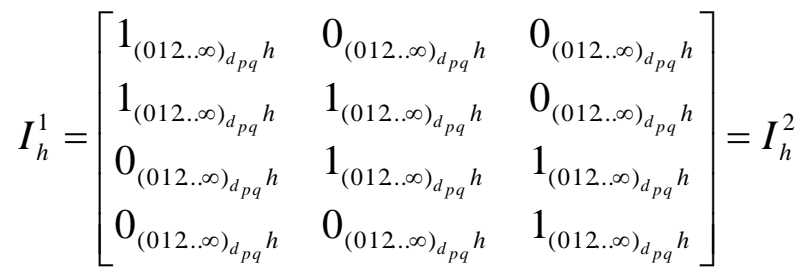

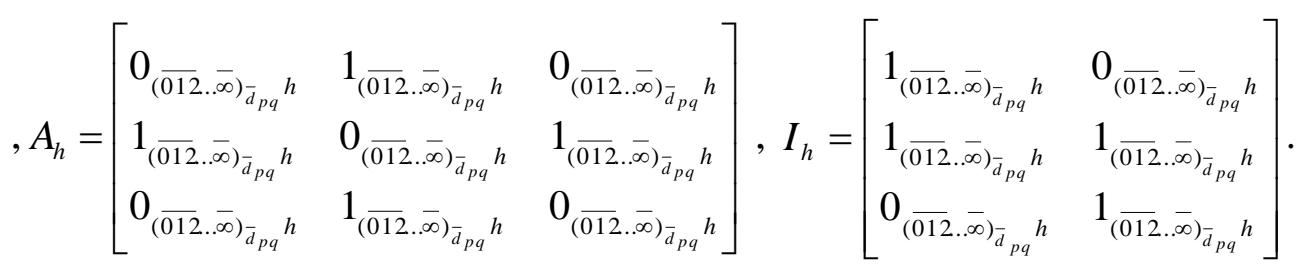

If any two chaotic graphs with density variation are intersected in one edge or in many edges, then both the adjacent and incidence matrices representing that intersection are not zero matrices and they are not sub matrices of those representing the given two chaotic graphs and the total 
density of the intersected graph will be reduced as it is a sub-graph of the two graphs, while it is increased in the intersected area.

\subsubsection{The intersection of two chaotic graphs with density variation common in more than one edge and vertex in another way:}

Consider two multiple chaotic graphs with density variation $\left\{e_{j h}^{i} ;\{i=1,2,3,5,8,9, j=0,1,2 \ldots\}\right\}$ and $G_{h}^{2}=\left\{v_{j h}^{i} ; i=\{1,2,3,4\} \&\{j=0,1,2,3, \ldots\}\right\} ; \quad\left\{e_{j h}^{i} ;\{i=2,3,4,5,6,7,8, j=0,1,2 \ldots\}\right\}$. See Figure (8).
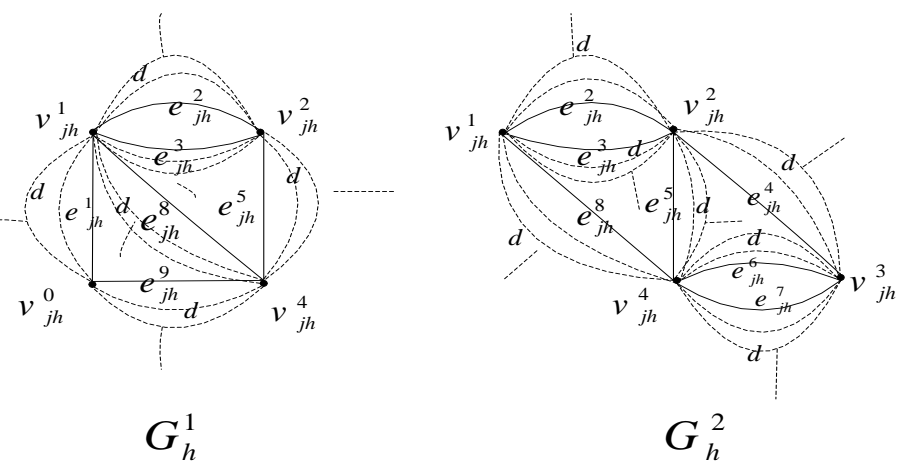

Figure (8): Intersection of two chaotic graphs common in more than one vertex and edge

The adjacent and incidence matrices of $G_{h}^{1}, G_{h}^{2}$ and $G_{h}^{1} \cap G_{h}^{2}$ are:

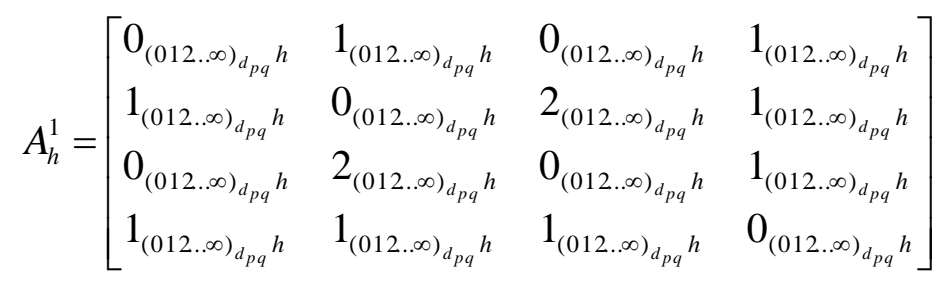




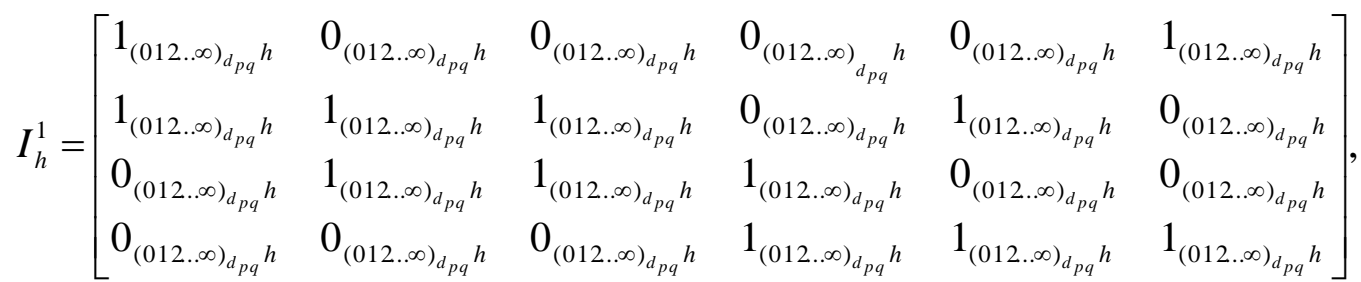

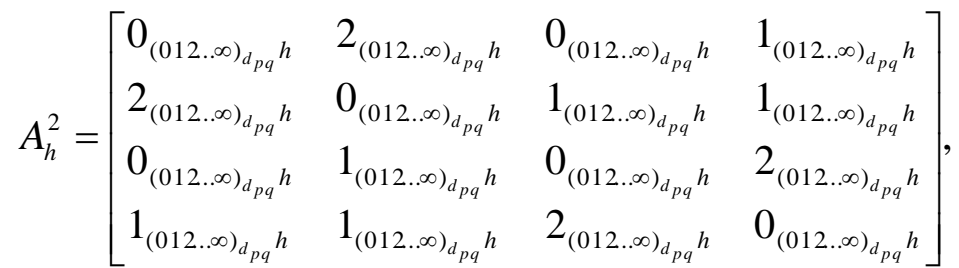

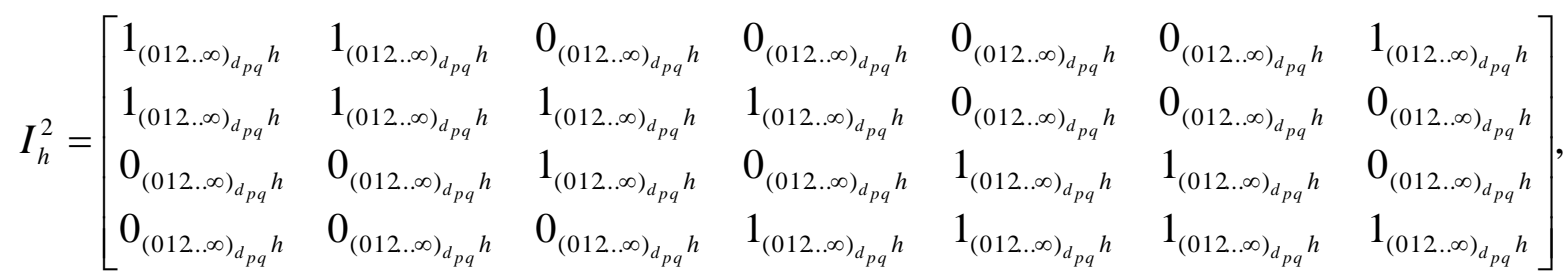

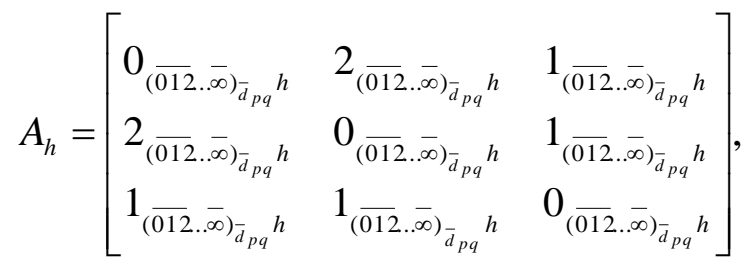

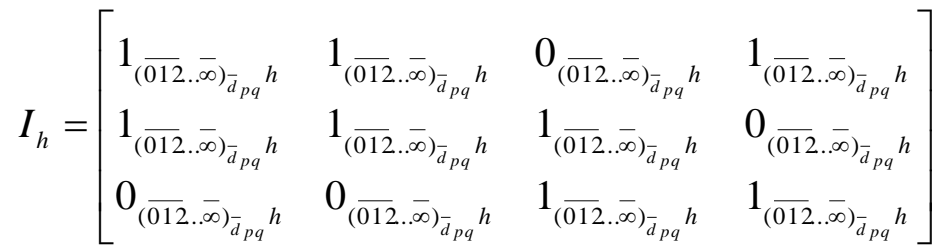

An important point is that both the adjacent and incidence matrices of the intersection of two multiple chaotic graphs are not sub matrices of those representing the given two multiple chaotic graph.

\section{Applications}

There are several applications applied on chaotic graphs with density variation such as the internet signal speeds and the variation of green color for different parts of the plant, for example, in botany, in some cases some plants suffer from a lack of chlorophyll, in this case the plant is represented by a chaotic graph and the proportion of chlorophyll is represented by the 
density property, and then the appropriate process is applied to increase the chlorophyll percentage in the appropriate place.

\section{Conclusions}

In this paper, the union and intersection of chaotic graphs with density variation was discussed. The union of two chaotic graphs result a new chaotic graph with more extremer density value. The intersection operation discussed in several cases, in all cases the density value will increase in the intersected area, but the total density of the resulted graph will be reduced compared to the original two graphs. If any two chaotic graphs with density variation are disjoint, then both the adjacent and incidence matrices representing that intersection are $\phi$-matrix, and the density value of the intersected graph is zero, while the adjacent and incidence matrices of the intersection of two chaotic graphs common in more than one vertex with density variation and without any common edges are zeros matrices, but they have a double density at the intersected chaotic vertices.

If any two chaotic graphs with density variation are intersected in more than one edge, then both the adjacent and incidence matrices representing that intersection are not zero matrices and they are not sub matrices of those representing the given two chaotic graphs such as the case of the intersection of two geometric graphs and the total density of the intersected graph will be reduced as it is a sub-graph of the two graphs, while it is increased in the intersected area.

The intersection of two chaotic graphs with density variation common in more than one edge and vertex resulted more extremer density in the intersected area as the total density will be reduced as it is a sub-graph of the intersected two graphs and both the adjacent and incidence matrices of the intersection of those graphs are not sub matrices of those representing the given two multiple chaotic graph. 
In future work, more advanced study on chaotic graphs will be discussed such as the unfolding transform and how it effects on the density value of the chaotic graph. Moreover; a comparison study will be discussed between the un-folding and folding transform which had been studied in previous paper.

\section{List of Abbreviations:}

\begin{tabular}{|l|l|}
\hline $\mathrm{d}$ & density \\
\hline$A_{h}(G)$ & Adjacency matrix of chaotic graph \\
\hline$I_{h}(G)$ & Incidence matrix of chaotic graph \\
\hline $\mathrm{h}$ & An index represents chaotic graph \\
\hline $\mathrm{G}$ & graph \\
\hline$G_{h}$ & Chaotic graph \\
\hline
\end{tabular}




\section{REFERENCES:}

[1] J.L. Gross, and T.W .Tucker, Topological Graph Theory, John Wiley \& Sons Inc. Canada 1987.

[2] M. El-Ghoul, A. El-Ahmady, and T. Homoda, On chaotic graphs and applications in physics and biology, Chaos Solutions and Fractals, 27, No.1, UK, January (2006) 159-173.

[3] M. EL-Ghoul, A. EI- Ahmady, and T. Homoda, Retraction of simplicial complexes, International Journal of Applied Mathematics and statistics, India, 4, No. J06 (2006) 54-67.

[4] A.Gibbons, Algorithmic graph theory, Cambridge University Press, Cambridge, UK, 1995.

[5] M. Abu-Saleem, Topological folding on the chaotic edge graphs and their fundamental group, International Journal of Pure and Applied Mathematics, 119, No.12, (2018)15479-15487.

[6] A. El-Ahmady, and H. M .Shamara, Fuzzy deformation retract of fuzzy horospheres,

Indian Journal of Pure \& Applied Mathematics, 32, No.10, October (2001)1501-1506.

[7] R.J. Wilson, Introduction to graph theory, Oliver \& Boyed, Edinburgh, 1972.

[8] S. Nada, and E.EL- Shafey, Chaotic graph an the sphere, British Journal of Mathematics \& Computer Science, 10(3), No.BJMCS.18791, January (2015) 1-27

[9] M. Abu-Saleem, Folding on the chaotic graph operations and their fundamental group, October 25th 2019, DOI: 10.5772/intechopen.88553.

[10] M. El-Ghoul ,H. Ahmed, and M. M. Khalil, Algorithm on tape graph and their geometric transformations, International Journal of Applied Science and Technology, 3,No.5,May (2013) 45-52.

[11] F. M. Alogab, Folding Simple chaotic graphs with density variation, Journal of Humanities and Applied Science (JHAS) ,2, No. 29, December (2016) 56-73. 